\title{
Transformation matrices for the Mueller-Jones formalism
}

\author{
Rafael Espinosa-Luna ${ }^{\mathrm{a}, \mathrm{b}}$, David Rodríguez-Carrera ${ }^{\mathrm{b}}$, \\ Eusebio Bernabeu ${ }^{\mathrm{a}, *}$, Sinhué Hinojosa-Ruíz ${ }^{\mathrm{c}}$ \\ a Applied Optics Complutense Group, Optics Department, Universidad Complutense de Madrid Facultad de Ciencias Fisicas, \\ Ciudad Universitaria s/n, 28040 Madrid, Spain \\ ${ }^{\mathrm{b}}$ Centro de Investigaciones en Optica, A.C., Loma del Bosque 115, Colonia Lomas del Campestre, 37150 León, \\ Guanajuato, México \\ ${ }^{\mathrm{c}}$ Facultad de Física, Universidad Autónoma de Zacatecas, Avenida Preparatoria 301, Fraccionamiento Progreso, \\ 28040 Zacatecas, Zac., México
}

Received 2 January 2007; accepted 28 March 2007

\begin{abstract}
The Mueller-Jones (MJ) or pure Mueller matrix formulation has been reported by using two different matrix transformations in a condensed representation. The possibility to find other transformation matrices is explored. A complete set of unitary operators $(R)$ is found to be closely related with the MJ matrices and with the evolution of pure states on the Poincaré sphere surface. We propose an alternative deduction for the condensed representation of the MJ matrices, obtained by using the Kronecker product operation and use of $R$ unitary matrices as a tool to combine different Mueller matrices and changes of polarized states on the Poincarè sphere surface. Finally, it is shown explicitly that the columns of the transformation matrices are the eigenvectors of the $\mathrm{MJ}$ matrix associated to a non-depolarizing optical system and a corollary is established as a criterion to differentiate a Mueller matrix from an MJ matrix.

(C) 2007 Elsevier GmbH. All rights reserved.
\end{abstract}

\section{Introduction}

It has been asserted that the Mueller matrix can give the full polarimetric characterization of a general scattering system. This matrix describes the modification of polarization of light after a linear interaction with the sample under interest is supposed. The use of the Mueller matrix formalism can be applied to nondepolarizing, partially or totally depolarizing systems,

\footnotetext{
*Corresponding author.

E-mail addresses: reluna@ cio.mx (R. Espinosa-Luna), ebernabeu@fis.ucm.es (E. Bernabeu).
}

as in the cases of rough surfaces and scattering media and for unpolarized, partially and totally polarized incident light, respectively. A Mueller-Jones (MJ) matrix has been defined as a Mueller matrix derived from a Jones matrix [1]. Some properties associated to the MJ matrix have been discussed in many works [1-9]. The MJ matrix has been derived from first principles and a condensed form to express it has been published [10-14]. Several authors have reported basically two forms for the transformation matrix employed into the condensed form $[5,6,12,13,15,16]$. These transformation matrices are related with the way the polarization of light is described. Commonly, they are used without any 
explicit consideration of the reference system employed for the description of the polarization; that is, looking to the source or to the propagating direction. We explore explicitly the possibility to find more transformation matrices through the analysis of 24 combinations for the representation of equivalent Stokes vectors. A set of unitary operators is found to be closely related with the MJ matrices and with the evolution of states on the Poincaré sphere surface. By applying the Kronecker product to the linear response of a physical system to an electric field, an alternative way to reach the condensed representation of the $\mathrm{MJ}$ is found. The $R$ unitary matrices are employed to obtain relations among Mueller matrices and/or $A$ transformation matrices $[5,6,12,13,15,16]$ and as a tool to describe changes of polarized states on the Poincarè sphere surface. Finally, it is shown explicitly that the columns of the transformation matrices are the eigenvectors of the MJ matrix associated to a non-depolarizing optical system and a corollary is established as a criterion to differentiate a Mueller matrix from an $\mathrm{MJ}$ matrix.

\section{Formulation of the Mueller-Jones matrix}

The linear response of a non-depolarizing physical system to an incident electric field $E^{\mathrm{inc}}$ is represented by the relation:

$E^{\mathrm{sc}}=J E^{\mathrm{inc}}$,

where $J$ is a $2 \times 2$ matrix of complex elements, named the Jones matrix of the system and $E^{\mathrm{sc}}$ is the scattered electric field. The electric fields are represented in terms of their orthogonal components by a $2 \times 1$ column matrix. On the other hand, the linear response of a physical system to polarization states can be expressed in terms of intensities (irradiances), through the relation:

$S^{\mathrm{sc}}=M S^{\mathrm{inc}}$,

where $M$ is called the Mueller matrix of the system, represented as a $4 \times 4$ matrix of real elements, and $S$ is a $4 \times 1$ matrix of real elements too, called the Stokes vector. $S$ represents the polarization state of the electric field vector, defined as $[12,15]$

$$
S^{\alpha}=\left(\begin{array}{c}
s_{1}^{\alpha} \\
s_{2}^{\alpha} \\
s_{3}^{\alpha} \\
s_{4}^{\alpha}
\end{array}\right),
$$

where $\alpha=$ inc, sc and

$$
\begin{aligned}
& s_{1}^{\alpha}=\left\langle E_{p}^{\alpha} E_{p}^{\alpha^{*}}\right\rangle+\left\langle E_{s}^{\alpha} E_{s}^{\alpha^{*}}\right\rangle, \\
& s_{2}^{\alpha}=\left\langle E_{p}^{\alpha} E_{p}^{\alpha^{*}}\right\rangle-\left\langle E_{\mathrm{s}}^{\alpha} E_{\mathrm{s}}^{\alpha^{*}}\right\rangle, \\
& s_{3}^{\alpha}=\left\langle E_{p}^{\alpha} E_{s}^{\alpha^{*}}\right\rangle+\left\langle E_{p}^{\alpha} E_{s}^{\alpha^{*}}\right\rangle^{*}=2 \operatorname{Re}\left\langle E_{p}^{\alpha} E_{s}^{\alpha^{*}}\right\rangle, \\
& s_{4}^{\alpha}=i\left(\left\langle E_{p}^{\alpha} E_{s}^{\alpha^{*}}\right\rangle-\left\langle E_{p}^{\alpha} E_{s}^{\alpha^{*}}\right\rangle^{*}\right)=-2 \operatorname{Im}\left\langle E_{p}^{\alpha} E_{s}^{\alpha^{*}}\right\rangle .
\end{aligned}
$$

Angular brackets represent ensemble averages and * indicates the complex conjugate operation. In this case, the polarization states are described as looking to the source [12].

\section{Transformation matrices for the Mueller-Jones matrices}

Eq. (3) can be expressed as a function of the electric field components through the matrix representation [12], which we propose can be ordered in the following way:

$$
\begin{aligned}
&\left(\begin{array}{l}
s_{1} \\
s_{2} \\
s_{3} \\
s_{4}
\end{array}\right)= {\left[\begin{array}{cccc}
1 & 0 & 0 & 1 \\
1 & 0 & 0 & -1 \\
0 & 1 & 1 & 0 \\
0 & i & -i & 0
\end{array}\right]\left(\begin{array}{c}
\left\langle E_{p} E_{p}^{*}\right\rangle \\
\left\langle E_{p} E_{s}^{*}\right\rangle \\
\left\langle E_{s} E_{p}^{*}\right\rangle \\
\left\langle E_{s} E_{s}^{*}\right\rangle
\end{array}\right) } \\
& \equiv A\left(\begin{array}{c}
\left\langle E_{p} E_{p}^{*}\right\rangle \\
\left\langle E_{p} E_{s}^{*}\right\rangle \\
\left\langle E_{s} E_{p}^{*}\right\rangle \\
\left\langle E_{s} E_{s}^{*}\right\rangle
\end{array}\right) \equiv\left[\begin{array}{llll}
a & b & c & d
\end{array}\right]\left[\begin{array}{c}
e \\
f \\
g \\
h
\end{array}\right],
\end{aligned}
$$

where $A$ takes on the form of one of the reported transformation matrices for the MJ matrix [5]. In this work, columns of the transformation matrix are represented by letters $a, b, c, d$ and rows of electric field products by letters $e, f, g, h$, respectively. By changing pairs of columns and the corresponding pairs of rows, 24 explicit combinations can arise for the representation 
of the Stokes vector, Eq. (5),

$$
\begin{aligned}
& {\left[\begin{array}{llll}
a & b & c & d
\end{array}\right]\left[\begin{array}{l}
e \\
f \\
g \\
h
\end{array}\right] ; \quad\left[\begin{array}{llll}
a & b & d & c
\end{array}\right]\left[\begin{array}{l}
e \\
f \\
h \\
g
\end{array}\right] ; \quad\left[\begin{array}{llll}
a & c & b & d
\end{array}\right]\left[\begin{array}{l}
e \\
g \\
f \\
h
\end{array}\right] ; \quad\left[\begin{array}{llll}
a & c & d & b
\end{array}\right]\left[\begin{array}{l}
e \\
g \\
h \\
f
\end{array}\right] ;} \\
& {\left[\begin{array}{llll}
a & d & b & c
\end{array}\right]\left[\begin{array}{l}
e \\
h \\
f \\
g
\end{array}\right] ; \quad\left[\begin{array}{llll}
a & d & c & b
\end{array}\right]\left[\begin{array}{l}
e \\
h \\
g \\
f
\end{array}\right]} \\
& {\left[\begin{array}{llll}
b & a & c & d
\end{array}\right]\left[\begin{array}{l}
f \\
e \\
g \\
h
\end{array}\right] ; \quad\left[\begin{array}{llll}
b & a & d & c
\end{array}\right]\left[\begin{array}{l}
f \\
e \\
h \\
g
\end{array}\right] ; \quad\left[\begin{array}{llll}
b & c & a & d
\end{array}\right]\left[\begin{array}{l}
f \\
g \\
e \\
h
\end{array}\right] ; \quad\left[\begin{array}{llll}
b & c & d & a
\end{array}\right]\left[\begin{array}{l}
f \\
g \\
h \\
e
\end{array}\right] \text {; }} \\
& {\left[\begin{array}{llll}
b & d & a & c
\end{array}\right]\left[\begin{array}{l}
f \\
h \\
e \\
g
\end{array}\right] ; \quad\left[\begin{array}{llll}
b & d & c & a
\end{array}\right]\left[\begin{array}{l}
f \\
h \\
g \\
e
\end{array}\right]} \\
& {\left[\begin{array}{llll}
c & a & b & d
\end{array}\right]\left[\begin{array}{l}
g \\
e \\
f \\
h
\end{array}\right] ; \quad\left[\begin{array}{llll}
c & a & d & b
\end{array}\right]\left[\begin{array}{l}
g \\
e \\
h \\
f
\end{array}\right] ; \quad\left[\begin{array}{llll}
c & b & a & d
\end{array}\right]\left[\begin{array}{l}
g \\
f \\
e \\
h
\end{array}\right] ; \quad\left[\begin{array}{llll}
c & b & d & a
\end{array}\right]\left[\begin{array}{l}
g \\
f \\
h \\
e
\end{array}\right] \text {; }} \\
& {\left[\begin{array}{llll}
c & d & a & b
\end{array}\right]\left[\begin{array}{l}
g \\
h \\
e \\
f
\end{array}\right] ; \quad\left[\begin{array}{llll}
c & d & b & a
\end{array}\right]\left[\begin{array}{l}
g \\
h \\
f \\
e
\end{array}\right]} \\
& {\left[\begin{array}{llll}
d & a & b & c
\end{array}\right]\left[\begin{array}{l}
h \\
e \\
f \\
g
\end{array}\right] ; \quad\left[\begin{array}{llll}
d & a & c & b
\end{array}\right]\left[\begin{array}{l}
h \\
e \\
g \\
f
\end{array}\right] ; \quad\left[\begin{array}{llll}
d & b & a & c
\end{array}\right]\left[\begin{array}{l}
h \\
f \\
e \\
g
\end{array}\right] ; \quad\left[\begin{array}{llll}
d & b & c & a
\end{array}\right]\left[\begin{array}{l}
h \\
f \\
g \\
e
\end{array}\right] \text {; }} \\
& {\left[\begin{array}{llll}
d & c & a & b
\end{array}\right]\left[\begin{array}{l}
h \\
g \\
e \\
f
\end{array}\right] ; \quad\left[\begin{array}{llll}
d & c & b & a
\end{array}\right]\left[\begin{array}{l}
h \\
g \\
f \\
e
\end{array}\right] \text {. }}
\end{aligned}
$$

From a mathematical point of view, we can say all the 24 matrices $A$ displayed into Eq. (6) are equivalent in the sense they give the same Stokes vector provided the orthogonal components of the electric field follows the order as specified by their associated matrices. The order given by $a, d, b, c$ and $e, h, f, g$ has been reported for the representation of the Stokes vector [14]. The Stokes vector has been described under another reference system, for example, the rotated system described by Schmieder [17]. From analyzing Eq. (6), a basic question 
emerges: are all the matrices $A$ transformation matrices in the sense they are associated to MJ matrices? We will build an answer in the following paragraphs. From Eq. (6), we will take special attention to the following representations for the Stokes vector: where $J$ is the Jones matrix, $\otimes$ denotes the Kronecker matrix product or direct product, $A^{-1}$ denotes the inverse matrix of $A, *$ is the complex conjugate and $\mathrm{T}$ denotes the transpose operation. Basically two different forms for matrix $A$ have been considered

$$
\begin{aligned}
& S=\left(\begin{array}{c}
s_{1} \\
s_{2} \\
s_{3} \\
s_{4}
\end{array}\right)=\left[\begin{array}{llll}
a & c & b & d
\end{array}\right]\left[\begin{array}{c}
e \\
g \\
f \\
h
\end{array}\right]=\left[\begin{array}{cccc}
1 & 0 & 0 & 1 \\
1 & 0 & 0 & -1 \\
0 & 1 & 1 & 0 \\
0 & -i & i & 0
\end{array}\right]\left(\begin{array}{c}
\left\langle E_{p} E_{p}^{*}\right\rangle \\
\left\langle E_{s} E_{p}^{*}\right\rangle \\
\left\langle E_{p} E_{s}^{*}\right\rangle \\
\left\langle E_{s} E_{s}^{*}\right\rangle
\end{array}\right) \equiv A_{2}\left(\begin{array}{c}
\left\langle E_{p} E_{p}^{*}\right\rangle \\
\left\langle E_{s} E_{p}^{*}\right\rangle \\
\left\langle E_{p} E_{s}^{*}\right\rangle \\
\left\langle E_{s} E_{s}^{*}\right\rangle
\end{array}\right), \\
& S=\left(\begin{array}{c}
s_{1} \\
s_{2} \\
s_{3} \\
s_{4}
\end{array}\right)=\left[\begin{array}{llll}
a & d & b & c
\end{array}\right]\left[\begin{array}{c}
e \\
h \\
f \\
g
\end{array}\right]=\left[\begin{array}{cccc}
1 & 1 & 0 & 0 \\
1 & -1 & 0 & 0 \\
0 & 0 & 1 & 1 \\
0 & 0 & i & -i
\end{array}\right]\left(\begin{array}{c}
\left\langle E_{p} E_{p}^{*}\right\rangle \\
\left\langle E_{s} E_{s}^{*}\right\rangle \\
\left\langle E_{p} E_{s}^{*}\right\rangle \\
\left\langle E_{s} E_{p}^{*}\right\rangle
\end{array}\right) \equiv A_{3}\left(\begin{array}{c}
\left\langle E_{p} E_{p}^{*}\right\rangle \\
\left\langle E_{s} E_{s}^{*}\right\rangle \\
\left\langle E_{p} E_{s}^{*}\right\rangle \\
\left\langle E_{s} E_{p}^{*}\right\rangle
\end{array}\right), \\
& S=\left(\begin{array}{c}
s_{1} \\
s_{2} \\
s_{3} \\
s_{4}
\end{array}\right)=\left[\begin{array}{llll}
b & a & d & c
\end{array}\right]\left[\begin{array}{c}
f \\
e \\
h \\
g
\end{array}\right]=\left[\begin{array}{cccc}
0 & 1 & 1 & 0 \\
0 & 1 & -1 & 0 \\
1 & 0 & 0 & 1 \\
i & 0 & 0 & -i
\end{array}\right]\left(\begin{array}{c}
\left\langle E_{p} E_{s}^{*}\right\rangle \\
\left\langle E_{p} E_{p}^{*}\right\rangle \\
\left\langle E_{s} E_{s}^{*}\right\rangle \\
\left\langle E_{s} E_{p}^{*}\right\rangle
\end{array}\right) \equiv A_{4}\left(\begin{array}{c}
\left\langle E_{p} E_{s}^{*}\right\rangle \\
\left\langle E_{p} E_{p}^{*}\right\rangle \\
\left\langle E_{s} E_{s}^{*}\right\rangle \\
\left\langle E_{s} E_{p}^{*}\right\rangle
\end{array}\right), \\
& S=\left(\begin{array}{c}
s_{1} \\
s_{2} \\
s_{3} \\
s_{4}
\end{array}\right)=\left[\begin{array}{llll}
c & d & a & b
\end{array}\right]\left[\begin{array}{c}
g \\
h \\
e \\
f
\end{array}\right]=\left[\begin{array}{cccc}
0 & 1 & 1 & 0 \\
0 & -1 & 1 & 0 \\
1 & 0 & 0 & 1 \\
-i & 0 & 0 & i
\end{array}\right]\left(\begin{array}{c}
\left\langle E_{s} E_{p}^{*}\right\rangle \\
\left\langle E_{s} E_{s}^{*}\right\rangle \\
\left\langle E_{p} E_{p}^{*}\right\rangle \\
\left\langle E_{p} E_{s}^{*}\right\rangle
\end{array}\right) \equiv A_{5}\left(\begin{array}{c}
\left\langle E_{s} E_{p}^{*}\right\rangle \\
\left\langle E_{s} E_{s}^{*}\right\rangle \\
\left\langle E_{p} E_{p}^{*}\right\rangle \\
\left\langle E_{p} E_{s}^{*}\right\rangle
\end{array}\right), \\
& S=\left(\begin{array}{l}
s_{1} \\
s_{2} \\
s_{3} \\
s_{4}
\end{array}\right)=\left[\begin{array}{llll}
d & c & b & a
\end{array}\right]\left[\begin{array}{c}
h \\
g \\
f \\
e
\end{array}\right]=\left[\begin{array}{cccc}
1 & 0 & 0 & 1 \\
-1 & 0 & 0 & 1 \\
0 & 1 & 1 & 0 \\
0 & -i & i & 0
\end{array}\right]\left(\begin{array}{c}
\left\langle E_{s} E_{s}^{*}\right\rangle \\
\left\langle E_{s} E_{p}^{*}\right\rangle \\
\left\langle E_{p} E_{s}^{*}\right\rangle \\
\left\langle E_{p} E_{p}^{*}\right\rangle
\end{array}\right) \equiv A_{6}\left(\begin{array}{c}
\left\langle E_{s} E_{s}^{*}\right\rangle \\
\left\langle E_{s} E_{p}^{*}\right\rangle \\
\left\langle E_{p} E_{s}^{*}\right\rangle \\
\left\langle E_{p} E_{p}^{*}\right\rangle
\end{array}\right) .
\end{aligned}
$$

The Mueller matrix obtained from Eq. (2) when the relation given by Eq. (1) holds, is a special kind of matrix because it can be obtained from a Jones matrix (it is well known that all Jones matrices can be described as Mueller matrices, but not all Mueller matrices can be derived from Jones matrices). Those kinds of matrices have been called non-depolarizing, pure or MJ matrices, respectively, if the system does not depolarize [1-16]. There does exists a condensed expression to define an MJ matrix, which has been reported as [1]

$$
M=A\left(J \otimes J^{*}\right) A^{-1} ; \quad A^{-1}=\frac{1}{2}\left(A^{\mathrm{T}}\right)^{*},
$$

$[5,6,11,12,15,16]$. These transformation matrices are related with the reference system employed to describe the polarization states. When polarization is described looking to the source, $A$ takes on the form $[12,15]$

$$
A=\left[\begin{array}{cccc}
1 & 0 & 0 & 1 \\
1 & 0 & 0 & -1 \\
0 & 1 & 1 & 0 \\
0 & i & -i & 0
\end{array}\right] \equiv A_{1},
$$


$A^{-1}=\frac{1}{2}\left[\begin{array}{cccc}1 & 1 & 0 & 0 \\ 0 & 0 & 1 & -i \\ 0 & 0 & 1 & i \\ 1 & -1 & 0 & 0\end{array}\right] \equiv A_{1}^{-1}$

But if polarization is described as looking to the propagation direction, $A$ is written as $[11,16]$

$$
\begin{aligned}
A= & {\left[\begin{array}{cccc}
1 & 0 & 0 & 1 \\
1 & 0 & 0 & -1 \\
0 & 1 & 1 & 0 \\
0 & -i & i & 0
\end{array}\right] \equiv A_{2}, } \\
A^{-1}= & \frac{1}{2}\left[\begin{array}{cccc}
1 & 1 & 0 & 0 \\
0 & 0 & 1 & i \\
0 & 0 & 1 & -i \\
1 & -1 & 0 & 0
\end{array}\right] \equiv A_{2}^{-1},
\end{aligned}
$$

Recently, Borovoi et al. [9] have reported a matrix $A$ that follows Eq. (8); a priori we will consider it as another transformation matrix. This matrix has been normalized to be a unitary transformation [9]

$A=\frac{1}{\sqrt{2}}\left[\begin{array}{cccc}1 & 0 & 0 & 1 \\ 1 & 0 & 0 & -1 \\ 0 & -1 & -1 & 0 \\ 0 & -i & i & 0\end{array}\right] \equiv A_{\mathrm{B}}$
$A=\frac{1}{\sqrt{2}}\left[\begin{array}{cccc}1 & 1 & 0 & 0 \\ 0 & 0 & -1 & i \\ 0 & 0 & -1 & -i \\ 1 & -1 & 0 & 0\end{array}\right] \equiv A_{\mathrm{B}}^{-1}$.

These transformation matrices are strongly associated to specific electric field products, corresponding to defined coordinate systems of reference from which the polarization is described. We propose here a possible representation for the Stokes vector in terms of matrix $A_{\mathrm{B}}$ can be the following:

$$
\begin{aligned}
& S_{\mathrm{B}}=\frac{1}{\sqrt{2}}\left[\begin{array}{cccc}
1 & 0 & 0 & 1 \\
1 & 0 & 0 & -1 \\
0 & -1 & -1 & 0 \\
0 & -i & i & 0
\end{array}\right] \sqrt{2}\left(\begin{array}{c}
\left\langle E_{p} E_{p}^{*}\right\rangle \\
-\left\langle E_{P} E_{s}^{*}\right\rangle \\
-\left\langle E_{s} E_{p}^{*}\right\rangle \\
\left\langle E_{s} E_{s}^{*}\right\rangle
\end{array}\right) \\
& =\frac{1}{\sqrt{2}}\left[\begin{array}{llll}
a & -b & -c & d
\end{array}\right] \sqrt{2}\left(\begin{array}{c}
e \\
-f \\
-g \\
h
\end{array}\right)=\left(\begin{array}{c}
s_{1} \\
s_{2} \\
s_{3} \\
s_{4}
\end{array}\right) \text {. }
\end{aligned}
$$

We propose the following methodology. By considering

$$
J=\left[\begin{array}{ll}
J_{1} & J_{4} \\
J_{3} & J_{2}
\end{array}\right],
$$

and substituting into Eq. (8), using matrices $A_{1}, A_{2}, A_{3}$, $A_{4}, A_{5}, A_{6}$ and $A_{\mathrm{B}}$ as transformation matrices, respectively, we obtain the explicit relations for the Mueller matrix

$$
\begin{aligned}
M_{1}= & {\left[\begin{array}{llll}
m_{11} & m_{12} & m_{13} & m_{14} \\
m_{21} & m_{22} & m_{23} & m_{24} \\
m_{31} & m_{32} & m_{33} & m_{34} \\
m_{41} & m_{42} & m_{43} & m_{44}
\end{array}\right] ; \quad M_{2}=\left[\begin{array}{cccc}
m_{11} & m_{12} & m_{13} & -m_{14} \\
m_{21} & m_{22} & m_{23} & -m_{24} \\
m_{31} & m_{32} & m_{33} & -m_{34} \\
-m_{41} & -m_{42} & -m_{43} & m_{44}
\end{array}\right] } \\
M_{3}= & {\left[\begin{array}{llll}
J_{1}\left(J_{1}^{*}+J_{2}^{*}+J_{3}^{*}+J_{4}^{*}\right) & J_{1}\left(J_{1}^{*}-J_{2}^{*}+J_{3}^{*}-J_{4}^{*}\right) & J_{4}\left(J_{1}^{*}+J_{2}^{*}+J_{3}^{*}+J_{4}^{*}\right) & -i J_{4}\left(J_{1}^{*}-J_{2}^{*}+J_{3}^{*}-J_{4}^{*}\right) \\
J_{1}\left(J_{1}^{*}-J_{2}^{*}-J_{3}^{*}+J_{4}^{*}\right) & J_{1}\left(J_{1}^{*}+J_{2}^{*}-J_{3}^{*}-J_{4}^{*}\right) & J_{4}\left(J_{1}^{*}-J_{2}^{*}-J_{3}^{*}+J_{4}^{*}\right) & -i J_{4}\left(J_{1}^{*}+J_{2}^{*}-J_{3}^{*}-J_{4}^{*}\right) \\
J_{3}\left(J_{1}^{*}+J_{2}^{*}+J_{3}^{*}+J_{4}^{*}\right) & J_{3}\left(J_{1}^{*}-J_{2}^{*}+J_{3}^{*}-J_{4}^{*}\right) & J_{2}\left(J_{1}^{*}+J_{2}^{*}+J_{3}^{*}+J_{4}^{*}\right) & -i J_{2}\left(J_{1}^{*}-J_{2}^{*}+J_{3}^{*}-J_{4}^{*}\right) \\
i J_{3}\left(J_{1}^{*}-J_{2}^{*}-J_{3}^{*}+J_{4}^{*}\right) & i J_{3}\left(J_{1}^{*}+J_{2}^{*}-J_{3}^{*}-J_{4}^{*}\right) & i J_{2}\left(J_{1}^{*}-J_{2}^{*}-J_{3}^{*}+J_{4}^{*}\right) & J_{2}\left(J_{1}^{*}+J_{2}^{*}-J_{3}^{*}-J_{4}^{*}\right)
\end{array}\right] ; } \\
M_{4}= & {\left[\begin{array}{cccc}
m_{33} & i m_{34} & m_{31} & -i m_{32} \\
-i m_{43} & m_{44} & -i m_{41} & -m_{42} \\
m_{31} & i m_{14} & m_{11} & -i m_{12} \\
i m_{23} & -m_{24} & i m_{21} & m_{22}
\end{array}\right] ; \quad M_{5}=\left[\begin{array}{cccc}
i m_{43} & m_{44} & i m_{41} & -m_{42} \\
m_{13} & -i m_{14} & m_{11} & i m_{12} \\
-i m_{23} & -m_{24} & -i m_{21} & m_{22}
\end{array}\right] ; } \\
M_{6}= & {\left[\begin{array}{cccc}
m_{11} & -m_{12} & m_{13} & -m_{14} \\
-m_{21} & m_{22} & -m_{23} & m_{24} \\
m_{31} & -m_{32} & m_{33} & -m_{34} \\
m_{41} & -m_{42} & -m_{43} & m_{44}
\end{array}\right] ; \quad M_{\mathrm{B}}=\left[\begin{array}{cccc}
m_{11} & m_{12} & -m_{13} & -m_{14} \\
m_{21} & m_{22} & -m_{23} & -m_{24} \\
-m_{31} & -m_{32} & m_{33} & m_{34} \\
-m_{41} & -m_{42} & m_{43} & m_{44}
\end{array}\right], }
\end{aligned}
$$


where the MJ elements are given explicitly as [13]

$$
\begin{array}{ll}
m_{11}=\frac{1}{2}\left(\left|J_{1}\right|^{2}+\left|J_{2}\right|^{2}+\left|J_{3}\right|^{2}+\left|J_{4}\right|^{2}\right) ; \\
m_{12}=\frac{1}{2}\left(\left|J_{1}\right|^{2}-\left|J_{2}\right|^{2}+\left|J_{3}\right|^{2}-\left|J_{4}\right|^{2}\right) ; \\
m_{13}=\operatorname{Re}\left(J_{1} J_{4}^{*}+J_{2} J_{3}^{*}\right) ; \quad m_{14}=\operatorname{Im}\left(J_{1} J_{4}^{*}+J_{3} J_{2}^{*}\right) ; \\
m_{21}=\frac{1}{2}\left(\left|J_{1}\right|^{2}-\left|J_{2}\right|^{2}-\left|J_{3}\right|^{2}+\left|J_{4}\right|^{2}\right) ; \\
m_{22}=\frac{1}{2}\left(\left|J_{1}\right|^{2}+\left|J_{2}\right|^{2}-\left|J_{3}\right|^{2}-\left|J_{4}\right|^{2}\right) ; \\
m_{23}=\operatorname{Re}\left(J_{1} J_{4}^{*}-J_{2} J_{3}^{*}\right) ; \quad m_{24}=\operatorname{Im}\left(J_{1} J_{4}^{*}+J_{2} J_{3}^{*}\right) ; \\
m_{31}=\operatorname{Re}\left(J_{1} J_{3}^{*}+J_{2} J_{4}^{*}\right) ; \quad m_{32}=\operatorname{Re}\left(J_{1} J_{3}^{*}-J_{2} J_{4}^{*}\right) ; \\
m_{33}=\operatorname{Re}\left(J_{1} J_{2}^{*}+J_{3} J_{4}^{*}\right) ; \quad m_{34}=\operatorname{Im}\left(J_{1} J_{2}^{*}+J_{3} J_{4}^{*}\right) ; \\
m_{41}=-\operatorname{Im}\left(J_{1} J_{3}^{*}+J_{4} J_{2}^{*}\right) ; \quad m_{42}=-\operatorname{Im}\left(J_{1} J_{3}^{*}+J_{2} J_{4}^{*}\right) ; \\
m_{43}=-\operatorname{Im}\left(J_{1} J_{2}^{*}+J_{4} J_{3}^{*}\right) ; \quad m_{44}=\operatorname{Re}\left(J_{1} J_{2}^{*}-J_{3} J_{4}^{*}\right) .
\end{array}
$$

\section{Unitary operators}

On the other hand, we propose a complete set of unitary operators $R$ can be written as

$$
\begin{aligned}
& R_{a}=\left[\begin{array}{llll}
1 & 0 & 0 & 0 \\
0 & 1 & 0 & 0 \\
0 & 0 & 1 & 0 \\
0 & 0 & 0 & 1
\end{array}\right] ; \quad R_{b}=\left[\begin{array}{cccc}
1 & 0 & 0 & 0 \\
0 & 1 & 0 & 0 \\
0 & 0 & 1 & 0 \\
0 & 0 & 0 & -1
\end{array}\right] ; \\
& R_{c}=\left[\begin{array}{cccc}
1 & 0 & 0 & 0 \\
0 & 1 & 0 & 0 \\
0 & 0 & -1 & 0 \\
0 & 0 & 0 & 1
\end{array}\right] ; \quad R_{d}=\left[\begin{array}{cccc}
1 & 0 & 0 & 0 \\
0 & -1 & 0 & 0 \\
0 & 0 & 1 & 0 \\
0 & 0 & 0 & 1
\end{array}\right] ;
\end{aligned}
$$

$$
S^{\mathrm{sc}}=\left\{\begin{array}{ccc}
R_{a} S & R_{b} S & R_{c} S \\
\left(\begin{array}{c}
s_{1} \\
s_{2} \\
s_{3} \\
s_{4}
\end{array}\right) & \left(\begin{array}{c}
s_{1} \\
s_{2} \\
s_{3} \\
-s_{4}
\end{array}\right) & \left(\begin{array}{c}
s_{1} \\
s_{2} \\
-s_{3} \\
s_{4}
\end{array}\right)
\end{array}\right.
$$

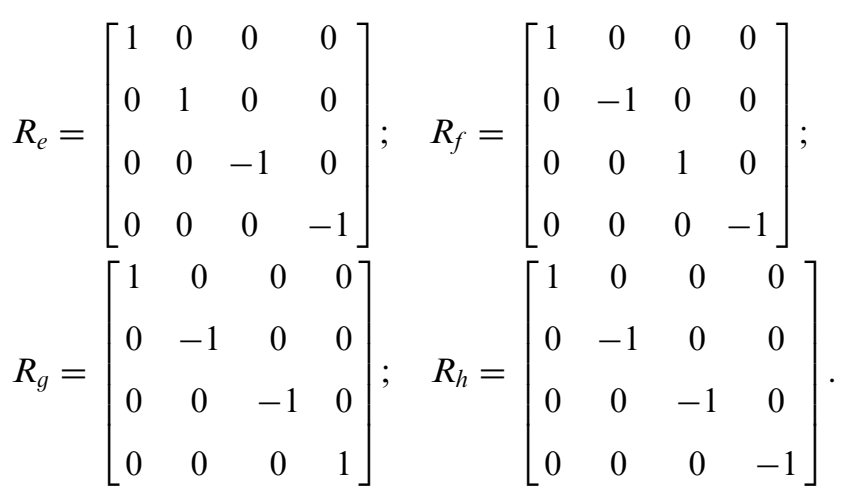

Note that an Abelian group can be obtained from Eq. (15). It can be easily verified that $M_{1}$ and $M_{2}$ are related through the unitary operator $R_{b}$

$M_{1}=R_{b} M_{2} R_{b}^{-1} \quad$ or $\quad M_{2}=R_{b} M_{1} R_{b}^{-1}$.

It follows that

$A_{1}=R_{b} A_{2}$.

On the other hand, $M_{1}$ and $M_{6}$ are related by

$M_{1}=R_{f} M_{6} R_{f}^{-1} \quad$ or $\quad M_{6}=R_{f} M_{1} R_{f}^{-1}$.

It follows that

$A_{1}=R_{f} A_{6}$.

Finally, $M_{1}$ and $M_{\mathrm{B}}$ are given as

$M_{1}=R_{\mathrm{e}} M_{\mathrm{B}} R_{\mathrm{e}}^{-1} \quad$ or $\quad M_{\mathrm{B}}=R_{\mathrm{e}} M_{1} R_{\mathrm{e}}^{-1}$,

and

$A_{1}=R_{\mathrm{e}} A_{\mathrm{B}}$.

We can say that not only the reported matrices $A_{1}, A_{2}$ and $A_{\mathrm{B}}$ are transformation matrices in the sense they generate an MJ matrix of real elements, but also $A_{6}$ is a transformation matrix. Note that $M_{3}, M_{4}$ and $M_{5}$ are complex matrices. If we do a similar exercise, we can verify that all the remaining matrices $A_{n}, n=7, \ldots, 24$ of Eq. (6) are not transformation matrices also.

What can we say about the physical significance of the unitary operators $R_{u}, u=a, \ldots, h$ ? Starting from the basic principle that an operator can be understood in the way it does affect a given function, we can apply $R_{u}$ to a general Stokes vector $S=\left(\begin{array}{llll}s_{1} & s_{2} & s_{3} & s_{4}\end{array}\right)^{\mathrm{T}}$.

$\begin{array}{ccccc}R_{d} S & R_{e} S & R_{f} S & R_{g} S & R_{h} S\end{array}$

$\left.\begin{array}{c}s_{1} \\ -s_{2} \\ s_{3} \\ s_{4}\end{array}\right) \quad\left(\begin{array}{c}s_{1} \\ s_{2} \\ -s_{3} \\ -s_{4}\end{array}\right) \quad\left(\begin{array}{c}s_{1} \\ -s_{2} \\ s_{3} \\ -s_{4}\end{array}\right) \quad\left(\begin{array}{c}s_{1} \\ -s_{2} \\ -s_{3} \\ s_{4}\end{array}\right) \quad\left(\begin{array}{c}s_{1} \\ -s_{2} \\ -s_{3} \\ -s_{4}\end{array}\right)$.

Observe the unitary operator $R^{b}$ acts as a switch on circular polarization states, because it changes right- to left-handed circular polarization and conversely. On the other hand, it does not affect the linear polarization states. Then $R b$ can be employed as a transformation rule to describe polarization states as observed looking to the source or looking into the propagation direction. Another interpretation can be done in terms of the Poincaré sphere: the operator maps the north pole on the south pole, and conversely. Some of the unitary matrices given by Eq. (15) have been employed by different authors and they have interpreted them as reflectors $[19,20]$, among other interpretations. Special 
attention has been dedicated to $R_{h}[8,13,15]$; Grier Parke III considered it as a transforming matrix from a righthanded to a left-handed coordinate system [15]. However, none of these authors have reported the complete set of unitary matrices given by Eq. (15) and no one of them have interpreted it in the way we are reporting here.

Following a similar procedure, it can be found that the different unitary operators, Eq. (15), change the polarization states to other states contained on the Poincaré sphere surface. It is an interesting exercise to think about the physical elements required to simulate the effect on the Stokes vectors due to the unitary operators.

On the other hand, if we obtain the MJ matrix from first principles [5], Eq. (2), using Eqs. (3) and (4), but we use two different descriptions for the orthogonal components of the electric field vector, Eq. (1),

$$
\left(\begin{array}{l}
E_{p}^{\mathrm{sc}} \\
E_{s}^{\mathrm{sc}}
\end{array}\right)=\left[\begin{array}{ll}
J_{1} & J_{4} \\
J_{3} & J_{2}
\end{array}\right]\left(\begin{array}{l}
E_{p}^{\mathrm{inc}} \\
E_{s}^{\mathrm{inc}}
\end{array}\right) \Rightarrow\left\{\begin{array}{l}
E_{p}^{\mathrm{sc}}=J_{1} E_{p}^{\mathrm{inc}}+J_{4} E_{s}^{\mathrm{inc}} \\
E_{s}^{\mathrm{sc}}=J_{3} E_{p}^{\mathrm{inc}}+J_{2} E_{s}^{\mathrm{inc}}
\end{array}\right.
$$

and

$$
\left(\begin{array}{l}
E_{s}^{\mathrm{sc}} \\
E_{p}^{\mathrm{sc}}
\end{array}\right)=\left[\begin{array}{ll}
J_{1} & J_{4} \\
J_{3} & J_{2}
\end{array}\right]\left(\begin{array}{l}
E_{s}^{\mathrm{inc}} \\
E_{p}^{\mathrm{inc}}
\end{array}\right) \Rightarrow\left\{\begin{array}{l}
E_{s}^{\mathrm{sc}}=J_{1} E_{s}^{\mathrm{inc}}+J_{4} E_{p}^{\mathrm{inc}} \\
E_{p}^{\mathrm{sc}}=J_{3} E_{s}^{\mathrm{inc}}+J_{2} E_{p}^{\mathrm{inc}}
\end{array}\right.
$$

we obtain again $M_{1}$ when Eq. (20a) is used and by using Eq. (20b) the MJ matrix is given as $M_{6}$.

The condensed representation for the MJ matrix, Eq. (8), can be obtained from the following procedure. If we calculate the Kronecker matrix product [18] of Eq. (20a) with its complex conjugate,

$$
\left(\begin{array}{l}
E_{p}^{\mathrm{sc}} E_{p}^{\mathrm{sc} *} \\
E_{p}^{\mathrm{sc}} E_{s}^{\mathrm{sc} *} \\
E_{s}^{\mathrm{sc}} E_{p}^{\mathrm{sc} *} \\
E_{s}^{\mathrm{sc}} E_{s}^{\mathrm{sc} *}
\end{array}\right)=\frac{1}{2}\left[\begin{array}{cccc}
1 & 1 & 0 & 0 \\
0 & 0 & 1 & -i \\
0 & 0 & 1 & i \\
1 & -1 & 0 & 0
\end{array}\right]\left[\begin{array}{ll}
m_{11} & m_{12} \\
m_{21} & m_{22} \\
m_{31} & m_{32} \\
m_{41} & m_{42}
\end{array}\right.
$$

$$
\begin{aligned}
\left(\begin{array}{c}
E_{p}^{\mathrm{sc}} \\
E_{s}^{\mathrm{sc}}
\end{array}\right) \otimes\left(\begin{array}{c}
E_{p}^{\mathrm{sc}} \\
E_{s}^{\mathrm{sc}}
\end{array}\right)^{*}= & \left\{\left[\begin{array}{ll}
J_{1} & J_{4} \\
J_{3} & J_{2}
\end{array}\right]\left(\begin{array}{c}
E_{p}^{\mathrm{inc}} \\
E_{s}^{\mathrm{inc}}
\end{array}\right)\right\} \\
& \otimes\left\{\left[\begin{array}{ll}
J_{1} & J_{4} \\
J_{3} & J_{2}
\end{array}\right]\left(\begin{array}{c}
E_{p}^{\mathrm{inc}} \\
E_{s}^{\mathrm{inc}}
\end{array}\right)\right\} .
\end{aligned}
$$

Following elemental procedures of multiplication, a factoring process and an ensemble average, Eq. (21a) can be written as

$$
\begin{gathered}
\left(\begin{array}{c}
\left\langle E_{p}^{\mathrm{sc}} E_{p}^{\mathrm{sc} *}\right\rangle \\
\left\langle E_{p}^{\mathrm{sc}} E_{s}^{\mathrm{sc} *}\right\rangle \\
\left\langle E_{s}^{\mathrm{sc}} E_{p}^{\mathrm{sc} *}\right\rangle \\
\left\langle E_{s}^{\mathrm{sc}} E_{s}^{\mathrm{sc} *}\right\rangle
\end{array}\right)=\left[\begin{array}{llll}
J_{1} J_{1}^{*} & J_{1} J_{4}^{*} & J_{4} J_{1}^{*} & J_{4} J_{4}^{*} \\
J_{1} J_{3}^{*} & J_{1} J_{2}^{*} & J_{4} J_{3}^{*} & J_{4} J_{2}^{*} \\
J_{3} J_{1}^{*} & J_{3} J_{4}^{*} & J_{2} J_{1}^{*} & J_{2} J_{4}^{*} \\
J_{3} J_{3}^{*} & J_{3} J_{2}^{*} & J_{2} J_{3}^{*} & J_{2} J_{2}^{*}
\end{array}\right] \\
\times\left(\begin{array}{c}
\left\langle E_{p}^{\mathrm{inc}} E_{p}^{\mathrm{inc} *}\right\rangle \\
\left\langle E_{p}^{\mathrm{inc}} E_{s}^{\mathrm{inc} *}\right\rangle \\
\left\langle E_{s}^{\mathrm{inc}} E_{p}^{\mathrm{inc} *}\right\rangle \\
\left\langle E_{s}^{\mathrm{inc}} E_{s}^{\mathrm{inc} *}\right\rangle
\end{array}\right)=\left(J \otimes J^{*}\right)\left(\begin{array}{c}
\left\langle E_{p}^{\mathrm{inc}} E_{p}^{\mathrm{inc} *}\right\rangle \\
\left\langle E_{p}^{\mathrm{inc}} E_{s}^{\mathrm{inc} *}\right\rangle \\
\left\langle E_{s}^{\mathrm{inc}} E_{p}^{\mathrm{inc} *}\right\rangle \\
\left\langle E_{s}^{\mathrm{inc}} E_{s}^{\mathrm{inc} *}\right\rangle
\end{array}\right) .
\end{gathered}
$$

On the other hand, employing Eq. (2), with the Stokes vector defined by Eq. (5), we have

$$
\begin{aligned}
& \left(\begin{array}{l}
s_{1}^{\mathrm{sc}} \\
s_{2}^{\mathrm{sc}} \\
s_{3}^{\mathrm{sc}} \\
s_{4}^{\mathrm{sc}}
\end{array}\right)=\left[\begin{array}{cccc}
1 & 0 & 0 & 1 \\
1 & 0 & 0 & -1 \\
0 & 1 & 1 & 0 \\
0 & i & -i & 0
\end{array}\right]\left(\begin{array}{c}
E_{p}^{\mathrm{sc}} E_{p}^{\mathrm{sc} *} \\
E_{p}^{\mathrm{sc}} E_{s}^{\mathrm{sc} *} \\
E_{s}^{\mathrm{sc}} E_{p}^{\mathrm{sc} *} \\
E_{s}^{\mathrm{sc}} E_{s}^{\mathrm{sc} *}
\end{array}\right) \\
& =\left[\begin{array}{llll}
m_{11} & m_{12} & m_{13} & m_{14} \\
m_{21} & m_{22} & m_{23} & m_{24} \\
m_{31} & m_{32} & m_{33} & m_{34} \\
m_{41} & m_{42} & m_{43} & m_{44}
\end{array}\right]\left[\begin{array}{cccc}
1 & 0 & 0 & 1 \\
1 & 0 & 0 & -1 \\
0 & 1 & 1 & 0 \\
0 & i & -i & 0
\end{array}\right]\left(\begin{array}{l}
E_{p}^{\mathrm{inc}} E_{p}^{\mathrm{inc} *} \\
E_{p}^{\mathrm{inc}} E_{s}^{\mathrm{inc} *} \\
E_{s}^{\mathrm{inc}} E_{p}^{\mathrm{inc} *} \\
E_{s}^{\mathrm{inc}} E_{s}^{\mathrm{inc} *}
\end{array}\right) .
\end{aligned}
$$

Resolving for the scattered electric field components, we obtain:

$$
\left.\begin{array}{ll}
m_{13} & m_{14} \\
m_{23} & m_{24} \\
m_{33} & m_{34} \\
m_{43} & m_{44}
\end{array}\right]\left[\begin{array}{cccc}
1 & 0 & 0 & 1 \\
1 & 0 & 0 & -1 \\
0 & 1 & 1 & 0 \\
0 & i & -i & 0
\end{array}\right]\left(\begin{array}{l}
E_{p}^{\text {inc }} E_{p}^{\text {inc* }} \\
E_{p}^{\text {inc }} E_{s}^{\text {inc* }} \\
E_{s}^{\text {inc }} E_{p}^{\text {inc* }} \\
E_{s}^{\text {inc }} E_{s}^{\text {inc* }}
\end{array}\right) .
$$

After an ensemble average, Eq. (23a) can be written in a more condensed form as

$$
\left(\begin{array}{c}
\left\langle E_{p}^{\mathrm{sc}} E_{p}^{\mathrm{sc} *}\right\rangle \\
\left\langle E_{p}^{\mathrm{sc}} E_{s}^{\mathrm{sc} *}\right\rangle \\
\left\langle E_{s}^{\mathrm{sc}} E_{p}^{\mathrm{sc} *}\right\rangle \\
\left\langle E_{s}^{\mathrm{sc}} E_{s}^{\mathrm{sc} *}\right\rangle
\end{array}\right)=A^{-1} M A\left(\begin{array}{c}
\left\langle E_{p}^{\mathrm{inc}} E_{p}^{\mathrm{inc} *}\right\rangle \\
\left\langle E_{p}^{\mathrm{inc}} E_{s}^{\mathrm{inc} *}\right\rangle \\
\left\langle E_{s}^{\mathrm{inc}} E_{p}^{\mathrm{inc} *}\right\rangle \\
\left\langle E_{s}^{\mathrm{inc}} E_{s}^{\mathrm{inc} *}\right\rangle
\end{array}\right),
$$


where $A$ and $A^{-1}$ are given by Eq. (5) or (9) and $M$ is the Mueller matrix of the system. Note carefully that at this stage, the Mueller matrix of Eq. (23b) can be associated to any arbitrary linear physical medium. But, if we consider that Eqs. (21b) and (23b) describe the same system, we are imposing a restriction to the arbitrariness of selecting $M$ because we are selecting a special kind of system; in this case, a system represented by a Jones matrix. If this is the situation, then we can equate the factor of the incident electric field on both equations, to obtain:

$J \otimes J^{*}=A^{-1} M A \Rightarrow M=A\left(J \otimes J^{*}\right) A^{-1}$.

In this way we have arrived at the MJ matrix definition by following the procedure proposed by us in this work.

We can go further ahead on the transformation matrices nature and give another interpretation for the transformation matrix based on a basis for the Stokes vectors. For simplicity, we use a one-dimensional (1-D) rough surface as example; however, the result must be valid for any non-depolarizing system. A 1-D rough surface is described with respect to an orthogonal coordinate Cartesian system as a surface whose profile varies along the $x$-axis and is constant along the $y$-axis [21,22]. A 2-D rough surface is a surface whose profile varies along both orthogonal axes. The MJ matrix for a 1-D surface can be obtained from Eq. (13) when $J_{3}=$ $J_{4}=0[21,22]$ :

$M=\left[\begin{array}{cccc}S_{11} & S_{12} & 0 & 0 \\ S_{12} & S_{11} & 0 & 0 \\ 0 & 0 & S_{33} & S_{34} \\ 0 & 0 & -S_{34} & S_{33}\end{array}\right]$,

where

$$
\begin{gathered}
S_{11}=\frac{1}{2}\left(J_{1} J_{1}^{*}+J_{2} J_{2}^{*}\right), \\
S_{12}=\frac{1}{2}\left(J_{1} J_{1}^{*}-J_{2} J_{2}^{*}\right), \\
S_{33}=\frac{1}{2}\left(J_{1} J_{2}^{*}+\left(J_{1} J_{2}^{*}\right)^{*}\right)=\operatorname{Re}\left(J_{1} J_{2}^{*}\right), \\
S_{34}=-\frac{i}{2}\left(J_{1} J_{2}^{*}-\left(J_{1} J_{2}^{*}\right)^{*}\right)=\operatorname{Im}\left(J_{1} J_{2}^{*}\right) .
\end{gathered}
$$

Our contribution in this work is explained in the following paragraphs. The eingenvalues of the MJ matrix, $M$, can be determined by the characteristic equation

$\operatorname{det}(M-\lambda I)=0$,

where $I$ is the $4 \times 4$ identity matrix and $\lambda$ denotes the eigenvalues of $M$. Eq. (27) can be solved for the eigenvalues as

$$
\begin{aligned}
\lambda=\left\{\lambda_{1}\right. & =\left(S_{11}+S_{12}\right), \lambda_{2}=\left(S_{33}+\mathrm{i} S_{34}\right), \\
\lambda_{3} & \left.=\left(S_{33}-\mathrm{i} S_{34}\right), \lambda_{4}=\left(S_{11}-S_{12}\right)\right\} .
\end{aligned}
$$

The corresponding eigenvectors $\mathfrak{I}_{j}$ can be calculated from the relation

$\left(M-\lambda_{j} I\right) \mathfrak{I}_{j}=0 \quad$ for $\quad j=1,2,3,4$.

It follows that a particular set for the eigenvectors can be given explicitly as

$\mathfrak{J}=\left\{\left(\begin{array}{l}1 \\ 1 \\ 0 \\ 0\end{array}\right),\left(\begin{array}{l}0 \\ 0 \\ 1 \\ i\end{array}\right),\left(\begin{array}{c}0 \\ 0 \\ 1 \\ -i\end{array}\right),\left(\begin{array}{c}1 \\ -1 \\ 0 \\ 0\end{array}\right)\right\}$.

These eigenvectors are linearly independent. This ordered set can be interpreted as a basis for the $4 \times 1$ matrices of complex values. Stokes vectors can be expressed as linear combinations of the eigenvectors given by Eq. (30) weighted by appropriate complex coefficients. The Stokes vectors are a subset of the complex linear space constituted by $4 \times 1$ matrix elements, in this case a subset formed by real elements (this does not mean Eq. (30) is a basis for the 4 real parameters that define a Stokes vector). For example, a generic Stokes vector can be written as

$$
\begin{aligned}
S= & \left\langle E_{p} E_{p}^{*}\right\rangle\left(\begin{array}{l}
1 \\
1 \\
0 \\
0
\end{array}\right)+\left\langle E_{s} E_{s}^{*}\right\rangle\left(\begin{array}{c}
1 \\
-1 \\
0 \\
0
\end{array}\right)+\left\langle E_{p} E_{s}^{*}\right\rangle\left(\begin{array}{l}
0 \\
0 \\
1 \\
i
\end{array}\right) \\
& +\left\langle E_{s} E_{p}^{*}\right\rangle\left(\begin{array}{c}
0 \\
0 \\
1 \\
-i
\end{array}\right)=\left(\begin{array}{c}
\left\langle E_{p} E_{p}^{*}\right\rangle+\left\langle E_{s} E_{s}^{*}\right\rangle \\
\left\langle E_{p} E_{p}^{*}\right\rangle-\left\langle E_{s} E_{s}^{*}\right\rangle \\
\left\langle E_{p} E_{s}^{*}\right\rangle+\left\langle E_{s} E_{p}^{*}\right\rangle \\
i\left(\left\langle E_{p} E_{s}^{*}\right\rangle-\left\langle E_{s} E_{p}^{*}\right\rangle\right)
\end{array}\right) .
\end{aligned}
$$

Observe that this ordered set of eigenvectors, Eq. (30), can be interpreted as column elements of a transformation matrix into a space $4 \times 4$ of complex elements

$\mathfrak{I}=\left[\begin{array}{cccc}1 & 0 & 0 & 1 \\ 1 & 0 & 0 & -1 \\ 0 & 1 & 1 & 0 \\ 0 & i & -i & 0\end{array}\right]$ 
It follows that Eq. (31) can be written as

$$
S=\left(\begin{array}{c}
s_{1} \\
s_{2} \\
s_{3} \\
s_{4}
\end{array}\right)=\left[\begin{array}{cccc}
1 & 0 & 0 & 1 \\
1 & 0 & 0 & -1 \\
0 & 1 & 1 & 0 \\
0 & i & -i & 0
\end{array}\right]\left(\begin{array}{c}
\left\langle E_{p} E_{p}^{*}\right\rangle \\
\left\langle E_{p} E_{s}^{*}\right\rangle \\
\left\langle E_{s} E_{p}^{*}\right\rangle \\
\left\langle E_{s} E_{s}^{*}\right\rangle
\end{array}\right),
$$

which is the same as Eq. (5); this is consistent with previously reported results [12]. By changing the order of columns and rows into Eq. (33), we can obtain the same results given by Eq. (6) and by appropriate handling, Eq. (12) can also be obtained.

Note that the MJ matrix can be expressed in terms of the condensed expression, Eq. (32), as

$M=\mathfrak{I}\left(J \otimes J^{*}\right) \mathfrak{J}^{-1}$,

where $J$ is given by Eq. (13) and $\mathfrak{I} \equiv A$, Eq. (9).

From the former result we can generalize, a priori, and the following Corollary is proposed by us in this work: a Mueller matrix is an MJ matrix if its eigenvectors are given by the set defined by Eq. (30).

\section{Conclusions}

The MJ of pure Mueller matrix formulations have been reported by using two different matrix transformations in a condensed representation. By considering 24 equivalent representations for the Stokes vector, we have found that another transformation matrix exists. We have obtained the condensed representation of the MJ matrix by applying a Kronecker product between the expression for the linear response of a physical system described by a Jones matrix and its complex conjugate. We have found a set $R$ of unitary matrices which can be employed as a tool to obtain relations among Mueller matrices and/or $A$ transformation matrices and to describe changes of polarized states on the Poincarè sphere surface. It has been shown explicitly that the columns of the transformation matrices are the eigenvectors of the MJ matrix associated to a nondepolarizing optical system. Finally a generalization, $a$ priori, of the previous result has been proposed here as a Corollary that asserts a Mueller matrix is an MJ matrix if its eigenvectors are given by the set defined by Eq. (30).

\section{Acknowledgments}

One of the authors, R.E.L., expresses his gratitude to CONACYT (Project 46969-F), to CONCYTEG (05-04K117-066-A02) and to Grupo Santander (Program Visitantes Distinguidos at the Universidad Complutense de Madrid) for the support provided for the realization of this work.

\section{References}

[1] R. Barakat, Bilinear constraints between elements of the $4 \times 4$ Mueller-Jones transfer matrix of polarization optics, Opt. Commun. 38 (1981) 159-161.

[2] E.S. Fry, G.W. Kattawar, Relationships between elements of the Stokes matrix, Appl. Opt. 20 (1981) 2811-2814.

[3] R. Simon, The connection between Mueller and Jones matrices of polarization optics, Opt. Commun. 42 (1982) 293-297.

[4] J.J. Gil, E. Bernabeu, A depolarization criterion in Mueller matrices, Opt. Acta 32 (1985) 259-261.

[5] J.W. Hovenier, Structure of a general pure Mueller matrix, Appl. Opt. 33 (1994) 8318-8324.

[6] C. Brosseau, C.R. Givens, A.B. Kotinski, Generalized trace condition on the Mueller-Jones polarization matrix, JOSA Commun. 10 (1993) 2248-2251.

[7] R.E. Luna, S.E. Acosta-Ortiz, L.-F. Zou, Mueller matrix for characterization of one-dimensional rough perfectly reflecting surfaces in a conical configuration, Opt. Lett. 23 (1998) 1075-1077.

[8] J.J. Gil, Characteristic properties of Mueller matrices, J. Opt. Soc. Am. A 17 (2000) 328-334.

[9] A.G. Borovoi, I. Grishin, Scattering matrices for large ice crystal particles, J. Opt. Soc. Am. A 20 (2003) 2071-2080.

[10] H.C. van de Hulst, Light Scattering by Small Particles, Dover, New York, 1957.

[11] E.L. O'Neill, Introduction to Statistical Optics, AddissonWesley, Reading, Mass, 1963.

[12] R.M.A. Azzam, N.M. Bashara, Ellipsometry and Polarized Light, North-Holland, Amsterdam, 1989.

[13] C. Brosseau, Fundamentals of Polarized Light: Statistical Optics Approach, Wiley, New York, 1998.

[14] S. Huard, Polarisation de la Lumiere, Masson, Paris, 1994, 33pp.

[15] N. Grier, Parke III, Matrix Optics, Ph.D. Thesis, MIT Press, Cambridge, MA, 1948.

[16] E. Wolf, Il Nuovo Cimento, Ser 10, 13 (1959) 1165.

[17] R.W. Schmieder, Stokes - algebra formalism, J. Opt. Soc. Am. 59 (1969) 297-302.

[18] F.M. Kronz, J.T. Tiehen, Emergence and quantum mechanics, Philos. Sci. 69 (2002) 314-347.

[19] B.J. DeBoo, J.M. Sasian, R.A. Chipman, Depolarization of diffusely reflecting man-made objects, Appl. Opt. 44 (2005) 5434-5445.

[20] E. Wolfe, R.A. Chipman, Polarimetric characterization of liquid-crystal-on-silicon panels, Appl. Opt. 45 (2006) 1688-1703.

[21] K.A. O'Donnell, M.E. Knotts, Polarization dependence of scattering from one-dimensional rough surfaces, J. Opt. Soc. Am. A 8 (1991) 1126-1131.

[22] N.C. Bruce, A.J. Sant, J.C. Dainty, The Mueller matrix for rough surface scattering using the Kirchhoff approximation, Opt. Commun. 88 (1992) 471. 